\title{
Mecanismo de Frank Starling: una visión aplicada en la erección del pene
}

\section{Frank-Starling Mechanism: A Vision Applied To Penile Erection}

\author{
Nathalia Buitrago Gómez ${ }^{1}$ Johnie Smith Husbands² \\ ${ }^{1}$ Médico, Universidad Libre, Cali, Valle del Cauca, Colombia \\ ${ }^{2}$ Anestesiólogo, Universidad del Valle, Universidad Libre, Cali, Valle \\ del Cauca, Colombia \\ ${ }^{3}$ Asociado Escuela de Medicina, Universidad del Valle, Director Grupo \\ de Investigación UROGIV y Grupo Asociado Cochrane, Cali, Valle del \\ Cauca, Colombia
}

\author{
Herney Andrés García-Perdomo 3
}

\begin{abstract}
Address for correspondence Nathalia Buitrago Gómez, MD, Universidad Libre de Cali, calle 44\#23 a 30, barrio nuevo príncipe, Tuluá - Valle, Colombia (e-mail: natybugo@gmail.com).
\end{abstract}

Urol Colomb 2019;28:183-188.

\section{Resumen}

\section{Palabras Clave}

- insuficiencia cardíaca

- erección peniana

- disfunción eréctil
El mecanismo de Frank-Starling es un concepto básico de la fisiología cardíaca y su aprendizaje. Constituye la base fundamental para el entendimiento del funcionamiento del corazón y patologías prevalentes y con alta morbimortalidad como lo es la insuficiencia cardíaca. Por lo cual, su relación con un proceso más interactivo como la erección del pene, podría asegurar un aprendizaje perdurable y práctico. La relación se fundamentó en el reemplazo del eje X de la curva por precarga en el caso del corazón y estimulación en el caso del pene, para así lograr una relación incluso con la disfunción eréctil, que sería el equivalente a la insuficiencia cardíaca. De esa manera, se encontró que a mayor estimulación hay mayor erección y a mayor precarga hay mayor eyección ventricular, teniendo ambas curvas una meseta. Asímismo, farmacológicamente, se encontró relación con el uso de estimulación $\beta$-adrenérgica y de inhibidores selectivos de la fosfodiesterasa tipo 5 como el sildenafil, los cuales desplazan la curva hacia arriba y a la izquierda.

The Frank-Starling mechanism is a basic concept of cardiac physiology and the fundamental basis for understanding the cardiac performance and prevalent disorders at risk of high morbidity and mortality, such as heart failure. Therefore, its relation with an interactive process like penile erection may enable deeper insight into cardiac physiology. The X-axis of Frank-Starling's curve was changed from ventricular end-diastolic volume to stimulation, to achieve a relation that includes erectile dysfunction, which is the equivalent of heart failure. A direct relationship was found between stimulation and erection, as well as end-diastolic volume and ventricular ejection with a plateau in both curves. Likewise, pharmacologically, a relation was identified with the use of $\beta$-adrenergic and selective inhibitor of phosphodiesterase (PDE) type 5 like sildenafil, which shift leftward and upward the Frank-Starling curve. received

November 27, 2017

accepted

April 6, 2018
DOI https://doi.org/

$10.1055 / \mathrm{s}-0038-1648238$.

ISSN 0120-789X.

eISSN 2027-0119.
Copyright $($ C 2019, Sociedad Colombiana License terms de Urología. Publicado por Thieme Revinter Publicações Ltda., Rio de Janeiro, Brazil. Todos los derechos reservados.

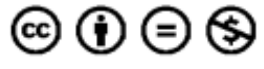




\section{Introducción}

El mecanismo de Frank-Starling, reconocido universalmente como una ley básica de la fisiología cardíaca, explica la forma de adaptación del corazón al cambio de flujo sanguíneo. Fue formulado en sus inicios como: "la energía total liberada en cada latido cardíaco es determinada por el volumen diastólico del corazón y por lo tanto, por la longitud de la fibra muscular al inicio de la contracción."1 Su aplicación favorece el entendimiento de patologías de gran relevancia y alta morbimortalidad como la insuficiencia cardíaca. Por consiguiente, facilitar su comprensión a través de una relación con un proceso cotidiano y científico como lo es la erección del pene, podría ayudar a un aprendizaje más eficaz. En primera instancia, es importante separar los dos temas para enfocarlos posteriormente como una unidad. El objetivo de este artículo, es describir la relación que puede existir entre el mecanismo de Frank Starling y la erección del pene, con la finalidad de lograr un aprendizaje a través de una relación interactiva.

\section{Fisiología Cardíaca y el Mecanismo de Frank Starling}

El músculo cardíaco funciona como un sincitio, es decir, que cuando se aplica un estímulo a alguna parte del músculo, resulta en la contracción del músculo entero. El proceso por el cual el potencial de acción de un miocito cardíaco permite la contracción, se denomina acoplamiento excitacióncontracción, en donde la onda de excitación se propaga rápidamente a lo largo del sarcolema permitiendo el influjo de calcio desde el intersticio a su vez que hay liberación de calcio desde el retículo sarcoplásmico. Ese calcio libre citosólico, activa la contracción de los miofilamentos (sístole). La diástole o relajación ocurre como resultado de la disminución de la concentración de calcio, mediante su entrada de nuevo al retículo sarcoplásmico y su salida por medio de la bomba $3 \mathrm{Na}-1 \mathrm{Ca}{ }^{2}$

La contractilidad del músculo cardíaco está influenciada por la precarga y la poscarga. El primer término se refiere a la fuerza que se extiende a lo largo de las fibras musculares relajadas, la cual se relaciona directamente con el volumen de fin de diástole. Mientras que, la poscarga hace referencia a la fuerza contra la cual, el músculo contraído debe actuar, que en el caso del ventrículo izquierdo sería la presión en la aorta. ${ }^{2}$

El descubrimiento de que el volumen de fin de diástole regula el trabajo del corazón es generalmente acreditado a Ernest Starling quien describió esa relación en 1912, y citó en su escrito que no fueron los únicos que hablaron de ella, dando crédito también a Otto Frank quien en 1895 lo estudió también. ${ }^{3}$ En ella se representa la longitud inicial de la fibra miocárdica o el volumen ventricular de fin de diástole y la fuerza desarrollada o presión ventricular. Esa ley es una propiedad intrínseca del miocardio, el cual, tiene la capacidad de incrementar la longitud de sus sarcómeros frente al incremento del retorno venoso o del volumen de fin de diástole, con la finalidad de aumentar a su vez el volumen sistólico. Es decir que, los ventrículos pueden acomodarse ante ese incremento de volumen mediante una contracción más vigorosa (-Fig. 1). ${ }^{3}$
Aunque ha sido una ley ampliamente reconocida, su base celular aún no es bien entendida. Una de las hipótesis más aceptadas es que el incremento lineal del sarcómero, incrementa la interacción entre los filamentos cruzados con actina, debido a que se reduce el espacio lateral existente entre filamentos delgados y gruesos, permitiendo que exista mayor proximidad entre ellos y por tanto, se incremente la fuerza contráctil. $^{4,5} \mathrm{Al}$ mismo tiempo, se ha descrito que el incremento de la fuerza inicial es debido primariamente a un aumento en la sensibilidad al calcio a nivel de los miofilamentos. ${ }^{6}$

Por tanto, un corazón normal intacto, opera en la porción ascendente de la curva de Frank-Starling, ya que, aunque usualmente la presión ventricular diastólica se encuentra entre 0 a $7 \mathrm{~mm} \mathrm{Hg}$ y la longitud promedio del sarcómero es de $2.2 \mu \mathrm{m}$, incluso ante una presión diastólica $>50 \mathrm{~mm} \mathrm{Hg}$, la longitud no sobrepasa los $2.6 \mu \mathrm{m}$; mostrando la habilidad del miocardio para resistir el estiramiento ante altas presiones de llenado, lo cual, puede ser un mecanismo de seguridad cuando exista sobrecarga en diástole. ${ }^{7}$

La curva de función ventricular que ellos plantearon, muestra inicialmente un ascenso rápido de la presión ventricular ante presiones diastólicas bajas, mientras que, ante presiones de fin de diástole altas, la curva se aplana hasta formar una meseta. Existe también una porción descendente (decremento del trabajo ventricular con incremento en la presión efectiva diastólica), lo cual en sus estudios no ocurría sobre perros normales pero si sobre el corazón de un miocardio disfuncional. $^{8}$

Por eso se pueden mostrar diferentes líneas que representen la relación de Frank-Starling (-Fig. 2). Ante eso, se ha visto de forma importante que esa relación en el estadio tardío de la insuficiencia cardíaca, se encuentra muy deprimida en algunos casos, llegando a ser hasta 10 veces menor. ${ }^{7}$ Esos hallazgos se han demostrado incluso in-vivo, en donde se ha evidenciado que el mecanismo de Frank-Starling en la insuficiencia cardíaca congestiva está agotado y es incapaz de responder ante un incremento de carga con un aumento de la contractilidad, probablemente por una disminución en el incremento de la sensibilización de la miofibrilla al calcio después del aumento de la longitud, demostrándose por esa razón, que esos pacientes operan en la meseta o en la parte final de la curva. ${ }^{9-11}$

\section{Fisiología de la Erección del Pene}

El pene es el órgano masculino que permite la micción y las relaciones sexuales. Se ha descrito que su anatomía posee muchas de las partes distintivas de un esqueleto hidrostático, entendiéndose esqueleto como cualquier sistema que transmite fuerzas y en el caso de hidrostático, va a funcionar con dos elementos: el fluido y una pared de tejido circundante reforzada con proteínas fibrosas, constituyendo una interacción crucial que le brinda soporte. Las dos estructuras hidrostáticas eréctiles son el cuerpo cavernoso y esponjoso. El cuerpo cavernoso se encuentra en la parte dorsal del pene y es un sistema de alta presión responsable del incremento del tamaño del pene y la rigidez a la flexión durante la erección. Está compuesto de un espacio vascular revestido por células 


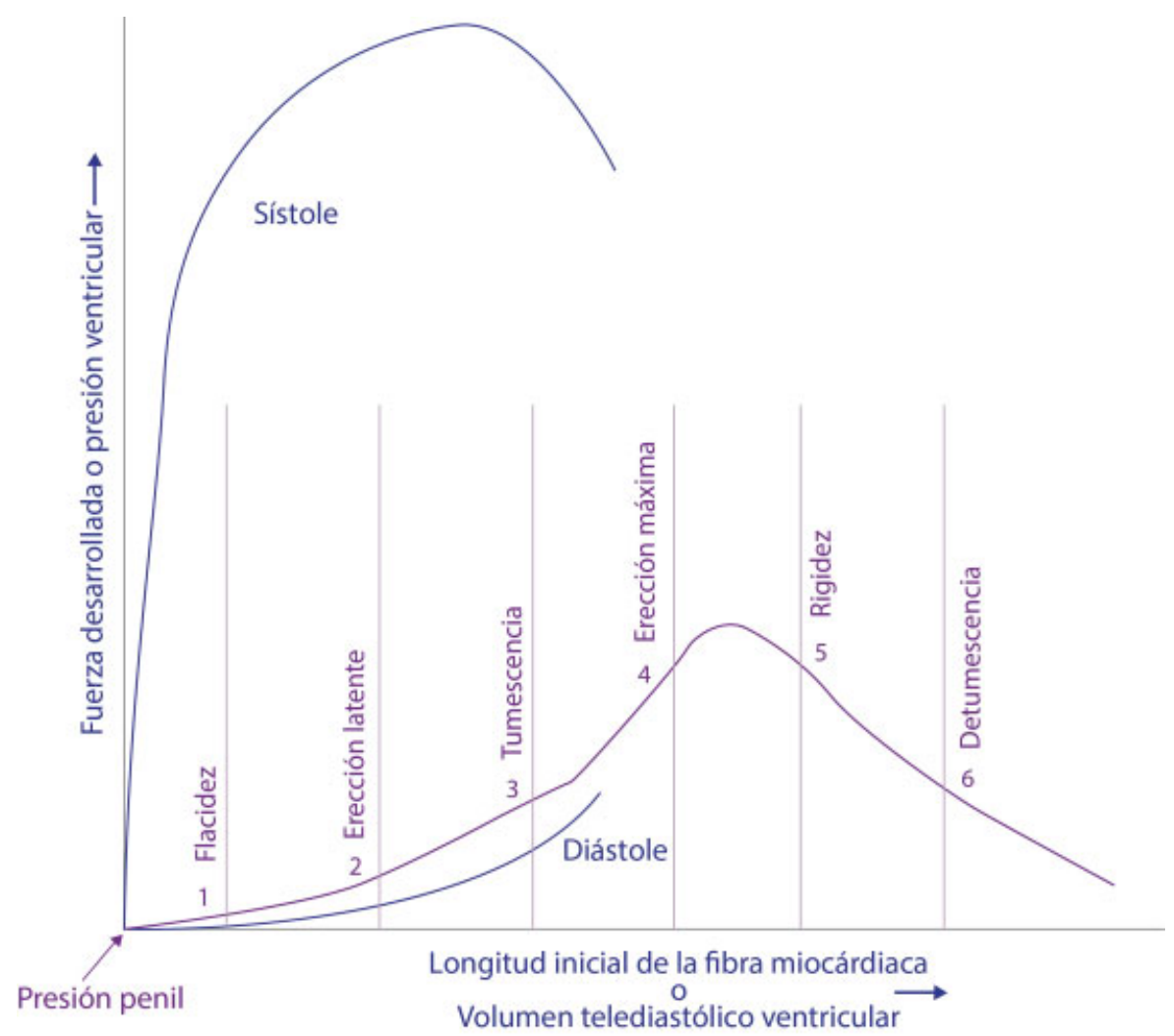

Fig. 1 En azul se representa la línea de Frank Starling y en morado el comportamiento de la presión penil en las distintas fases hemodinámicas de la erección del pene. En el gráfico, se logra relacionar el mecanismo de Frank Starling con uno de los parámetros hemodinámicos de la erección del pene (presión penil), en la cual, la estimulación logra un incremento en la misma, hasta llegar a la erección máxima y rigidez, en donde la forma de la curva simula la meseta del mecanismo de Frank Starling en el corazón.

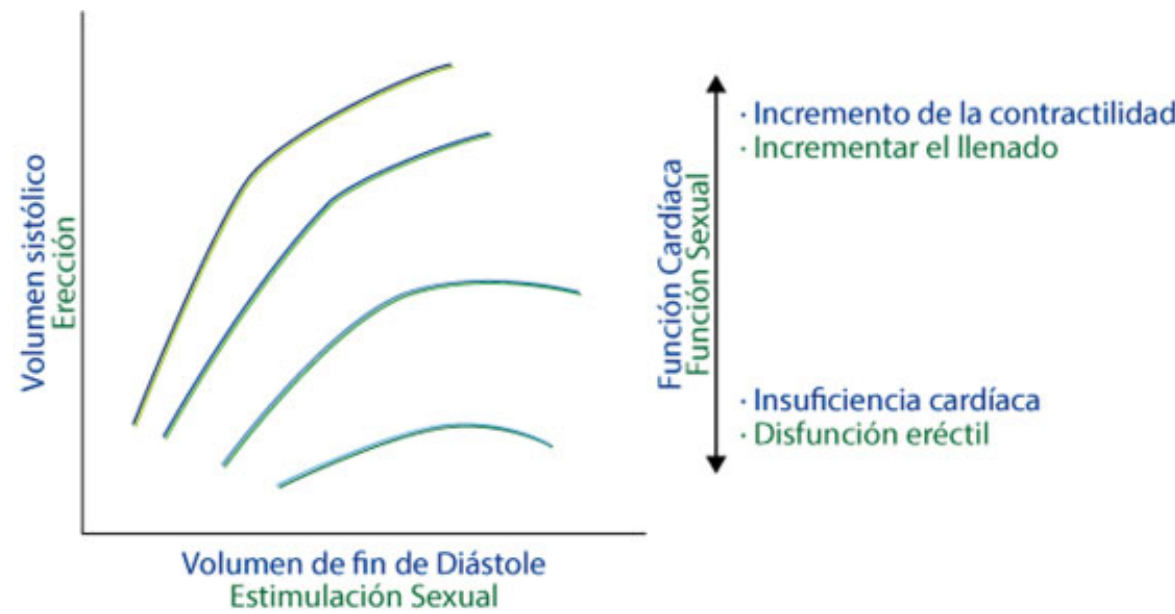

Fig. 2 Comparación de la respuesta de la erección y volumen sistólico, con base en la estimulación sexual y el volumen de fin de diástole respectivamente. En verde, se encuentra la representación de la función sexual mientras que, en azul, se encuentra la de la función cardíaca. La línea 2 muestra la relación de Frank-Starling en un corazón y un pene que funciona normalmente. Es decir, el incremento de la estimulación sexual y del volumen de fin de diástole, permiten una adecuación en la erección y en el volumen sistólico, con la finalidad de responder satisfactoriamente ante la demanda. Esa relación puede ser modulada hacia arriba (línea 1), mediante la implementación de estimulación $\beta$ adrenérgica o hacia abajo y hacia la derecha (línea 3 y 4), la cual ha sido definida como insuficiencia cardíaca o disfunción eréctil; en donde un incremento en el volumen de fin de diástole o en la estimulación sexual, resulta en un pequeño incremento en el volumen sistólico o la erección, inadecuado para satisfacer los requerimientos tisulares o lograr una relación sexual satisfactoria.

endoteliales, rodeado por una pared gruesa de tejido colágeno llamado túnica albugínea. El cuerpo esponjoso, más pequeño, contiene la uretra y se expande distalmente para formar el glande. A pesar de que también se somete a la vasodilatación durante la erección, no es responsable de la rigidez del pene. Cuando el pene es visto en sección transversal, se observa una 
parte central de tejido eréctil y esponjoso que se llena de fluido (sangre), y está rodeado por una pared de tejido rica en una proteína estructural denominada colágeno. ${ }^{12}$

En cuanto a la fisiología de la erección, resulta de la interacción de factores hormonales, vasculares, neurológicos, sinusoidales, musculares y psicológicos. Su principal controlador es el sistema nervioso autónomo, siendo ésta un fenómeno eminentemente neurovascular con un mediador universal que es el óxido nítrico. La vía simpática controla la erección por estimulación psicógena y el parasimpático sacro controla la erección reflexógena. Frente al estímulo sexual el flujo de las arterias cavernosas puede aumentar entre 20 a 50 veces. Por esa razón, el pene pasa de una presión intracavernosa en estado de flacidez que fluctúa entre 10 y $20 \mathrm{~mm} \mathrm{Hg}$ hasta $75 \mathrm{~mm} \mathrm{Hg}$ en la fase de tumescencia al iniciarse el proceso erectivo, e incluso llegando hasta 100 y $120 \mathrm{~mm} \mathrm{Hg}$ durante la erección en rigidez máxima. Durante la detumescencia se restablece la presión intracavernosa en forma gradual. ${ }^{13}$

Durante el proceso de la erección se reconocen 6 fases hemodinámicas diferentes. La primera, es la flacidez o estado basal en donde el pene está en reposo y el flujo arterial es bajo y suficiente para su nutrición. La segunda fase, es la erección latente o preerección, que requiere para su desarrollo algún tipo de estímulo (visual, táctil, auditivo, ligado al sueño) que active el proceso de la erección. El grado de erección es proporcional al grado de estimulación psíquico o físico. Comienza el estímulo neural por vía del nervio pudendo, para que el flujo peneano aumente intensamente, con una gran dilatación de la arteria y con aumento progresivo de la presión penil o intracavernosa.

La tercera fase es la tumescencia, en donde el estímulo fue exitoso para activar el proceso de la erección. El flujo peneano (FP) vía arteria pudenda disminuye un poco, con menor gasto de sangre para mantener la erección, existe disminución de la dilatación de la arteria y la presión intracavernosa comienza a bajar. A eso le sigue la cuarta fase o erección máxima, en donde en un inicio el flujo pudendo se estabiliza, al igual que la presión intracavernosa, la concentración de oxígeno en el abundante flujo sanguíneo es óptima y el proceso de venoclusión comienza a darse naturalmente. Posteriormente, el FP aumenta nuevamente, aunque la presión intracavernosa comienza a caer liberando el proceso de venoclusión. La concentración de oxígeno aumenta nuevamente.

Por su parte, en la quinta fase o rigidez, la arteria muestra el mínimo de dilatación. Con la venoclusión activada, el flujo sistólico cae, manteniendo la erección en su máximo de rigidez, la presión diastólica es cero, pero la concentración de oxígeno no es óptima por bajo flujo, que de perpetuarse sería de riesgo isquémico. Finalmente, en la última etapa que es la detumescencia, se da un disparo simpático que acompaña a la eyaculación o ha cesado el estímulo sexual, por lo que la erección empieza a caer; la venoclusión ya no es efectiva. La presión sistólica intracavernosa cae y la sangre nuevamente adquiere parámetros venosos (-Fig. $\mathbf{1}) .^{14}$

\section{Reflexión}

La erección del pene se puede inferir en la gráfica del mecanismo de Frank Starling. Es decir, cuando un hombre está sano, es capaz de responder adecuadamente ante la estimulación y a mayor llenado (precarga del pene), mayor erección. Para efectos de la relación, se debe poner en el eje $\mathrm{X}$ estimulación, puesto que la erección es directamente proporcional a la misma, y llega un momento donde entra en un período de meseta para mantener la relación sexual. Asímismo, si ese hombre se pone en contexto de una disfunción eréctil, se podría asemejar la gráfica a la insuficiencia cardíaca, donde la estimulación, por más amplia que sea, no genera una respuesta adecuada como para mantener la erección o lograr que una relación sexual sea satisfactoria. No sería acertado usar el volumen de fin de diástole que en el caso del pene sería el llenado de sangre, ya que en la disfunción, característicamente carece de ese componente de forma adecuada y por tal razón no se da la erección. Por tanto, usar la estimulación permite remontar la gráfica de disfunción eréctil, tomando en cuenta, que esos pacientes a pesar de tener la misma estimulación sexual, no responden de la misma forma (- Fig. 2).

Lo interesante a relacionar y observar es que, así como a mayor precarga en un corazón sano hay mayor eyección (presión ventricular); aquí en un pene sano, a mayor estimulación hay mayor llenado y con él, mayor erección (presión intracavernosa). Pero ¿qué pasaría con la meseta? Primero que todo, hay que observar que en el eje X no se está mencionando las fases del pene sino la estimulación sexual, diciendo que, ésta continúa aumentando durante la relación sexual, pero existe un momento en donde la estimulación es lo suficientemente alta como para que se dé el punto máximo de erección en una persona normal y su mantenimiento (meseta), que correspondería a la fase hemodinámica de erección máxima y rigidez, permite que la relación sexual sea satisfactoria hasta que se da el orgasmo y comienza la detumescencia y vuelve finalmente, cuando cesa la estimulación sexual, a su estado inicial de flacidez.

Ahora bien, así como la línea de Frank-Starling subía mediante el incremento de la contractilidad cardíaca, en ese caso sucede por el uso de $\beta$-adrenérgicos los cuales, aunque no se han usado como tal para mejorar la erección, de forma casual se ha demostrado que, los adrenoreceptores $\beta_{3}$ están presentes en el cuerpo cavernoso y localizados principalmente en las células musculares lisas, en donde su activación causa vasorelajación dependiente del GMPc (ya sea por su fosforilación, como por la inhibición de la vía RhoA/Rhoquinasa), y que el uso de antagonistas $\beta_{3}$-adrenérgico selectivos, causan efecto vasoconstrictor del cuerpo cavernoso dependiente de la concentración. ${ }^{15}$ Al mismo tiempo, se ha relacionado frecuentemente la terapia antihipertensiva con $\beta$-bloqueadores, como causa de disfunción eréctill, ${ }^{16}$ además de que se ha establecido como posible causa orgánica de ese padecimiento, la disfunción $\beta$-adrenérgica a nivel del pene. ${ }^{17}$ Es decir, que así como se dan $\beta$-adrenérgicos para elevar la gráfica de Frank Starling en el corazón, también se podrían dar para aumentarla en el pene, incluso ante una estimulación sexual menor.

La disfunción eréctil se define como la incapacidad para lograr y mantener una erección suficiente que permita un curso sexual satisfactorio. ${ }^{18} \mathrm{Al}$ mismo tiempo, la insuficiencia 
cardíaca es la incapacidad del corazón para lograr una eyección que permita cubrir los requerimientos metabólicos de los tejidos de forma satisfactoria. Por su parte, se puede observar en el gráfico, que así como en la insuficiencia cardíaca el corazón no tiene la capacidad de responder adecuadamente ante un incremento del volumen; en la disfunción eréctil el pene no es capaz de hacerlo ante un aumento de la estimulación sexual, o mejor dicho, la estimulación sexual no genera un adecuado llenado y erección como para lograr una relación sexual satisfactoria; en ese punto, se puede ver cómo su comportamiento sería similar. Por su parte, el sindenafil que es un inhibidor selectivo de la fosfodiesterasa tipo 5, la principal isoforma responsable de la hidrólisis del GMPc en la vasculatura pulmonar, ha demostrado que puede ser usado en la insuficiencia cardíaca sistólica, ya que, mejora la calidad de vida y la capacidad para hacer ejercicios. ${ }^{19,20}$ La importancia de mencionarlo en ese punto, es porque es un medicamento que funciona tanto en la insuficiencia cardíaca, como en la disfunción eréctil. Un mecanismo que de manera interesante puede ser mencionado y que corresponde al tema a tratar, es que se ha demostrado que el sildenafil incrementa la sensibilidad a la nitroglicerina, es decir, a la vasodilatación endotelio independiente mediada por óxido nítrico, ${ }^{21}$ que como ya se mencionó, es una de las moléculas principalmente involucradas en la erección. Asímismo, se ha demostrado que el óxido nítrico incrementa la sensibilidad de la respuesta al mecanismo de Frank Starling, ${ }^{22}$ por lo cual, el sildenafil podría funcionar incrementando la gráfica hacia arriba, tanto en el corazón, como a nivel del pene. Guazzi y col., en pacientes con insuficiencia cardíaca con fracción de eyección preservada, demostraron que el sildenafil genera desplazamiento hacia la izquierda de la relación de FrankStarling en el ventrículo derecho, lo cual produjo mejoría significativa en su funcionalidad. ${ }^{23}$

Al mismo tiempo, es tal su relación, que la insuficiencia cardíaca y disfunción eréctil, comparten factores de riesgo, causas y aparecen generalmente de forma concomitante. ${ }^{24-28}$ En la disfunción eréctil, se han descrito distintos factores etiológicos implicados, entre los que cabe resaltar, los endocrinológicos, en donde la diabetes se reconoce claramente como una causa de enfermedad cardiovascular ${ }^{29}$ y disfunción eréctil. ${ }^{30,31}$ Además, el hipogonadismo y su relación con enfermedad coronaria, ${ }^{32}$ insuficiencia cardíaca $^{33}$ y disfunción eréctil. ${ }^{34}$ Inclusive, múltiples estudios publicados han establecido a la disfunción eréctil como un marcador de riesgo independiente para la enfermedad cardiovascular, teniendo incluso un valor predictivo para eventos cardiovasculares similar a los factores de riesgo tradicionales. ${ }^{35}$

\section{Conclusiones}

La gráfica del mecanismo de Frank Starling, se puede aplicar tanto en el corazón como en el pene; usando incluso en una persona normal la misma variable en el eje X (precarga o volumen de llenado final), en donde, a mayor llenado va a existir mayor eyección en el caso del corazón y mayor erección en el caso del pene hasta llegar a una meseta; pero funcionando siempre en una persona sana, en la parte efectiva de la curva.

Para efectos prácticos, se podría cambiar el eje X en el caso del pene por estimulación sexual, con la finalidad de dibujar también la disfunción eréctil que sería en ese caso, la enfermedad concomitante con insuficiencia cardíaca puesto que, así como no responde un corazón en falla ante la precarga, tampoco un pene con disfunción ante la estimulación por más grande que la misma sea. Al mismo tiempo, farmacológicamente se encontró, que el efecto $\beta$ adrenérgico subiría la gráfica tanto en el corazón como en el pene o incluso, el sildenafil, desplaza hacia la izquierda la gráfica en ambos órganos.

Por su parte, la disfunción eréctil no sólo coincide con la insuficiencia cardíaca por su gráfica en el mecanismo de Frank Starling, sino que ambas tienden a aparecer de forma concomitante y comparten factores etiológicos como la diabetes y el hipogonadismo. De hecho, la disfunción eréctil se ha constituido en un factor independiente de riesgo cardiovascular por las similitudes que entre ambas existen, incluyendo la actualmente mencionada en esta relación.

\section{Responsabilidades Éticas}

Protección de personas y animales. Los autores declaran que para esta investigación no se han realizado experimentos en seres humanos ni en animales.

Confidencialidad de los datos. Los autores declaran que en este artículo no aparecen datos de pacientes.

Derecho a la privacidad y consentimiento informado. Los autores declaran que en este artículo no aparecen datos de pacientes.

\section{Conflicto de Intereses}

Los autores declaramos no tener ningún tipo de conflicto de intereses.

\section{Referencias}

1 Zimmer HG. Who discovered the Frank-Starling mechanism? News Physiol Sci 2002;17:181-184

2 Koeppen BM, Stanton BA. Berne \& Levy Physiology. Sixth edition; Elsevier2008

3 Katz AM. Ernest Henry Starling, his predecessors, and the "Law of the Heart". Circulation 2002;106(23):2986-2992 10.1161/01. CIR.0000040594.96123.55

4 Moss RL, Fitzsimons DP. Frank-Starling relationship: long on importance, short on mechanism. Circ Res 2002;90(01):11-13

5 Smith L, Tainter C, Regnier M, Martyn DA. Cooperative crossbridge activation of thin filaments contributes to the FrankStarling mechanism in cardiac muscle. Biophys J 2009;96(09): 3692-3702 10.1016/j.bpj.2009.02.018

6 Fuchs F, Smith SH. Calcium, cross-bridges, and the Frank-Starling relationship. News Physiol Sci 2001;16:5-10

7 Hanft LM, Korte FS, McDonald KS. Cardiac function and modulation of sarcomeric function by length. Cardiovasc Res 2008;77(04):627-636 10.1093/cvr/cvm099

8 Sarnoff SJ, Berglund E. Ventricular function. I. Starling's law of the heart studied by means of simultaneous right and left ventricular function curves in the dog. Circulation 1954;9(05):706-718 
9 Chan WL, Gilligan DM, Ang EL, Oakley CM. Effect of preload change on resting and exercise cardiac performance in hypertrophic cardiomyopathy. Am J Cardiol 1990;66(07):746-751

10 Gill RM, Jones BD, Corbly AK, et al. Exhaustion of the FrankStarling mechanism in conscious dogs with heart failure induced by chronic coronary microembolization. Life Sci 2006;79(06): 536-544 10.1016/j.lfs.2006.01.045

11 Schwinger RH, Böhm M, Koch A, et al. The failing human heart is unable to use the Frank-Starling mechanism. Circ Res 1994;74 (05):959-969

12 Kelly DA. Penises as variable-volume hydrostatic skeletons. Ann N Y Acad Sci 2007;1101:453-463 10.1196/annals.1389.014

13 Guyton AC, Hall JE. Tratado de fisiología médica. 11a ed. Elsevier; 2008

14 Uribe JF. Parámetros hemodinámicos en el proceso normal de la erección. Urol Colomb 2015;24:101-105 10.1016/j.uroco.2015. 05.011

15 Cirino G, Sorrentino R, di Villa Bianca Rd, et al. Involvement of beta 3-adrenergic receptor activation via cyclic GMP- but not NOdependent mechanisms in human corpus cavernosum function. Proc Natl Acad Sci U S A 2003;100(09):5531-5536 10.1073/ pnas.0931347100

16 Brixius K, Middeke M, Lichtenthal A, Jahn E, Schwinger RH. Nitric oxide, erectile dysfunction and beta-blocker treatment (MR NOED study): benefit of nebivolol versus metoprolol in hypertensive men. Clin Exp Pharmacol Physiol 2007;34(04): 327-331 10.1111/j.1440-1681.2007.04551.x

17 Ferini-Strambi L, Zucconi M, Rigatti P, Grasso M, Montorsi F, Smirne S. Male impotence: a possible beta-adrenergic dysfunction in some patients. Eur Urol 1992;21(04):332-334

18 Altinkilic B, Hauck EW, Weidner W. Evaluation of penile perfusion by color-coded duplex sonography in the management of erectile dysfunction. World J Urol 2004;22(05):361-364 10.1007/s00345004-0423-y

19 Blum A. Treating heart failure with sildenafil. Congest Heart Fail 2009;15(04):181-185 10.1111/j.1751-7133.2008.00026.x

20 Guazzi M. Sildenafil and phosphodiesterase-5 inhibitors for heart failure. Curr Heart Fail Rep 2008;5(02):110-114

21 Dishy V, Sofowora G, Harris PA, et al. The effect of sildenafil on nitric oxide-mediated vasodilation in healthy men. Clin Pharmacol Ther 2001;70(03):270-279 10.1067/mcp.2001.117995

22 Amelio D, Garofalo F, Capria C, Tota B, Imbrogno S. Effects of temperature on the nitric oxide-dependent modulation of the Frank-Starling mechanism: the fish heart as a case study. Comp
Biochem Physiol A Mol Integr Physiol 2013;164(02):356-362 10.1016/j.cbpa.2012.10.037

23 Guazzi M, Vicenzi M, Arena R, Guazzi MD. Pulmonary hypertension in heart failure with preserved ejection fraction: a target of phosphodiesterase-5 inhibition in a 1-year study. Circulation 2011;124(02):164-174 10.1161/CIRCULATIONAHA.110.983866

24 Rastogi S, Rodriguez JJ, Kapur V, Schwarz ER. Why do patients with heart failure suffer from erectile dysfunction? A critical review and suggestions on how to approach this problem. Int J Impot Res 2005;17(01, Suppl 1):S25-S36 10.1038/sj.ijir.3901426

25 Schwarz ER, Rastogi S, Kapur V, Sulemanjee N, Rodriguez JJ. Erectile dysfunction in heart failure patients. J Am Coll Cardiol 2006;48(06):1111-1119 10.1016/j.jacc.2006.05.052

26 Schwarz ER, Rodriguez J. Sex and the heart. Int J Impot Res 2005; 17(01, Suppl 1):S4-S6 10.1038/sj.ijir.3901422

27 Baraghoush A, Phan A, Willix RD Jr, Schwarz ER. Erectile dysfunction as a complication of heart failure. Curr Heart Fail Rep 2010;7(04):194-201 10.1007/s11897-010-0023-7

28 Andrianne R, D'Orio V. [Consensus on the clinical approach to erectile dysfunction in patients with cardiovascular disease]. Rev Med Liege 2000;55(05):454-458

29 Leon BM, Maddox TM. Diabetes and cardiovascular disease: Epidemiology, biological mechanisms, treatment recommendations and future research. World J Diabetes 2015;6(13):1246-1258 10.4239/wjd.v6.i13.1246

30 Carrier S, Brock G, Kour NW, Lue TF. Pathophysiology of erectile dysfunction. Urology 1993;42(04):468-481

31 Maiorino MI, Bellastella G, Esposito K. Diabetes and sexual dysfunction: current perspectives. Diabetes Metab Syndr Obes 2014;7:95-105 10.2147/DMSO.S36455

32 Morris PD, Channer KS. Testosterone and cardiovascular disease in men. Asian J Androl 2012;14(03):428-435 10.1038/aja.2012.21

33 Jankowska EA, Tkaczyszyn M, Węgrzynowska-Teodorczyk K, et al. Late-onset hypogonadism in men with systolic heart failure: prevalence, clinical associates, and impact on long-term survival. ESC Heart Fail 2014;1(01):41-51 10.1002/ehf2.12002

34 Dean RC, Lue TF. Physiology of penile erection and pathophysiology of erectile dysfunction. Urol Clin North Am 2005;32(04):379-395, v 10.1016/j.ucl.2005.08.007

35 Miner M, Seftel AD, Nehra A, et al. Prognostic utility of erectile dysfunction for cardiovascular disease in younger men and those with diabetes. Am Heart J 2012;164(01):21-28 10.1016/j. ahj.2012.04.006 